\title{
Peptide nucleic acids on microarrays and other biosensors
}

\author{
Ole Brandt and Jörg D. Hoheisel
}

Functional Genome Analysis, Deutsches Krebsforschungszentrum, Im Neuenheimer Feld 580, 69120 Heidelberg, Germany

The analysis of biomolecules using microarrays and other biosensors has a significant role in molecular biotechnology, and will become even more important in the future as a versatile tool for research and diagnostics. For many applications, the synthetic DNA mimic peptide nucleic acid (PNA) could be advantageous as a probe molecule, owing to its unique physicochemical and biochemical properties. PNA exhibits superior hybridization characteristics and improved chemical and enzymatic stability relative to nucleic acids. Furthermore, its different molecular structure enables new modes of detection, especially procedures that avoid the introduction of a label. In our opinion, all of these factors contribute significantly toward the establishment of faster and more reliable analytical processes and opens new fields of application.

Nucleic acids are a very frequently analyzed molecular class in current bioassays. The stability and specificity of the binding between DNA or RNA targets and supportbound probe molecules are crucial factors for the quality and robustness of the analyses. PNA is an entirely synthetic DNA mimic that was invented by Peter Nielsen et al. [1]. Its particular chemical properties make it suitable for use in bioassays. In PNA, the negatively charged (deoxy-) ribose-phosphate backbone of nucleic acids is replaced by an uncharged $\mathrm{N}$-(2-aminoethyl)glycine scaffold, to which the nucleobases are attached via a methylene carbonyl linker [1,2] (Figure 1). Because all intramolecular distances and the configuration of the nucleobases are similar to those in natural DNA molecules, specific hybridization occurs between PNA and cDNA or RNA sequences. The uncharged nature of PNA is responsible for a better thermal stability of PNA-DNA duplexes compared with the DNA-DNA equivalents. As a result, single-base mismatches have a considerably more destabilizing effect [2]. As with DNA, the decrease in duplex stability depends on the position of the mismatch within the sequence [3]. The neutral amide backbone also enables PNA to hybridize to DNA-molecules in low-salt conditions because no positive ions are necessary for counteracting the interstrand repulsion that hampers duplex formation between two negatively charged nucleic acids. Consequently, the abundance and stability of intramolecular folding structures in the DNA or RNA analytes are significantly reduced, making the molecules

Corresponding author: Ole Brandt (o.brandt@dkfz.de)

Available online 28 October 2004 more accessible to complementary PNA oligomers. Furthermore, PNA is stable across a wide range of temperatures and pHs, unlike DNA, which depurinates at acidic conditions. However, under alkaline conditions, a rearrangement of the PNA molecules might occur [4]. Additionally, PNA is resistant to nucleases and proteases [5]. For further reading about the affinity and specificity, as well as the chemical and biophysical properties of PNA molecules, we recommend two reviews [6,7].

Applications of PNA can be - in part arbitrarily - split into reactions that occur either in homogeneous solution [8] or with one interacting partner being attached to a solid support. The uses of PNA as antisense probe [9], DNA opener [10] or for polymerase chain reaction (PCR)

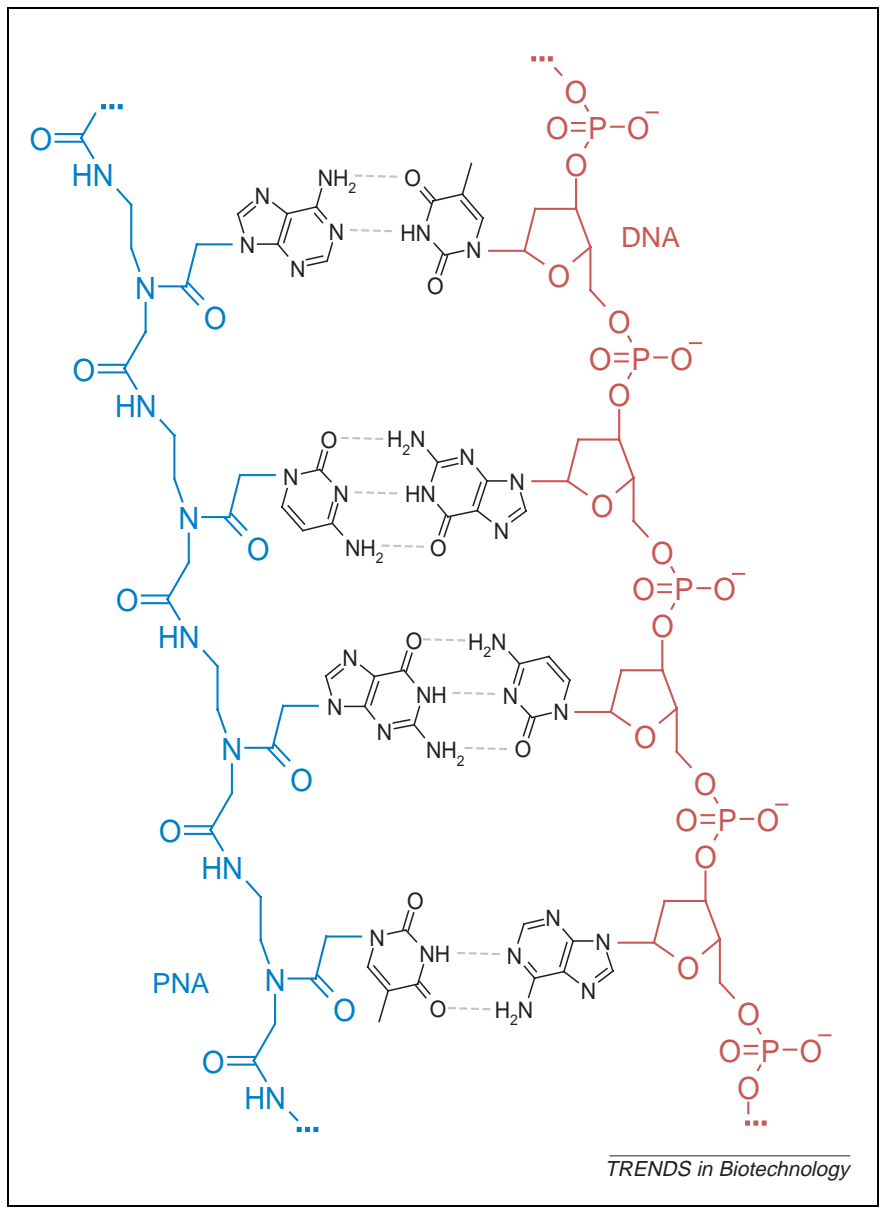

Figure 1. Comparison of the structures of peptide nucleic acid (PNA) and DNA. The deoxyribose-phosphate bacbone of DNA (red) is replaced by $\mathrm{N}$-(2-aminoethyl)glycine unite in PNA (blue). The nucleobases are shown in black. 
clamping [11,12] are important examples of the former format. Moreover, mutations and single nucleotide polymorphisms [13-15] have been detected in this way. Incorporation of PNA into electrophoresis matrices $[16,17]$ also falls into this class but represents an intermediate assay type because PNA is present in the gel matrix although not actually bound to it. Owing to its uncharged nature, it is not affected by the electric field and thereby remains stationary unless it interacts with a moving nucleic acid. In the second assay format, one partner - the PNA molecule or the complementary nucleic acid - is immobilized on a solid support. Microarrays made of PNA oligomers $[18,19]$ are typical examples. Assays of this latter analytical format are reviewed below. Although several assays are also performed using DNA-probes, PNA offers a wider variety in terms of its modes of action, especially with respect to detection. Either the samples being analyzed or the probe molecules can be labeled. However, the chemical differences of PNA compared with DNA make it possible to carry out direct, label-free detection of the interacting nucleic acids, which in turn could lead to fast and robust processes more suited for routine diagnostics such as those performed in a clinical environment.

\section{Investigation of labeled analytes}

Most analyses in this area are extensions of assays using DNA-probes. The substitution of PNA for DNA is considered to improve the quality of the assay, rather than introducing entirely new processes. However, for DNA microarrays, currently the most prominent assay format, the main advantage lies in the fact that a new means of detection becomes possible - and, in particular, one that avoids the labeling of the probe and the target altogether - and is therefore discussed below.

\section{Bead-based recovery and detection of nucleic acids}

A sequence-specific purification of particular nucleic acids is required for many purposes. One typical application of PNA in this process is the isolation of mRNA by means of magnetic beads that exhibit an oligo-T PNA-sequence at their surfaces. On incubation with nucleic acid isolates, mRNA molecules are captured by the strong interaction between their poly-A tails, and are separated from genomic DNA and rRNA by applying a magnetic field. Of course, molecules of more specific sequences can also be isolated. Chandler and Jarrell [20], for example, used labeled beads to capture and analyze 16S rRNA from different microorganisms. Each bead type has an internal color-code, by which it can be discriminated and thus counted in a flow cytometer. Total RNA was extracted, labeled with the fluorophore Alexa-532 and incubated with beads of different color, each color representing a particular PNA sequence. By dual excitation - one laser exciting the bead-specific fluorophore, the other activating Alexa-532 - the number of beads that interacted with individual rRNA-sequences, and thus the presence of different microorganisms, could be detected (Figure 2a). With a detection limit of $\sim 0.1 \mathrm{ng}$ total RNA, the assay was $\sim 100$ times more sensitive with PNA compared with DNA oligomers.

(a)
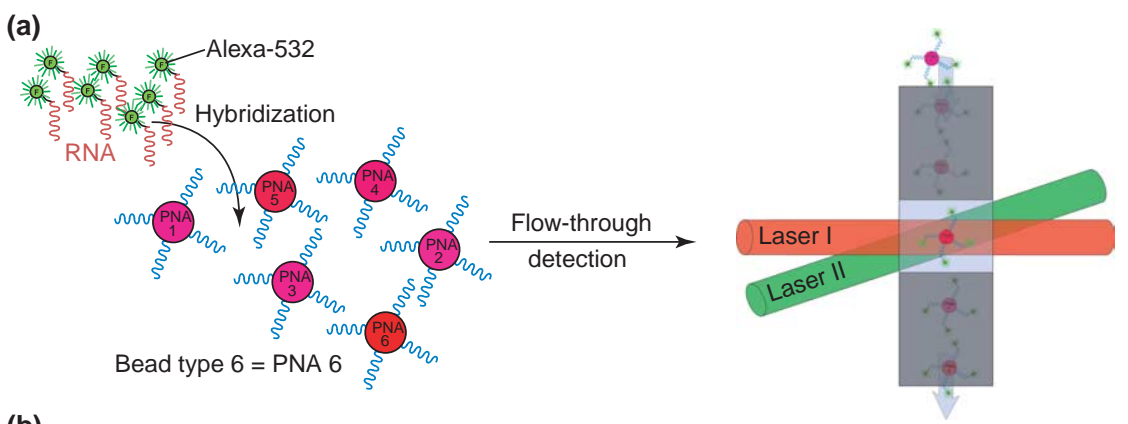

(b)

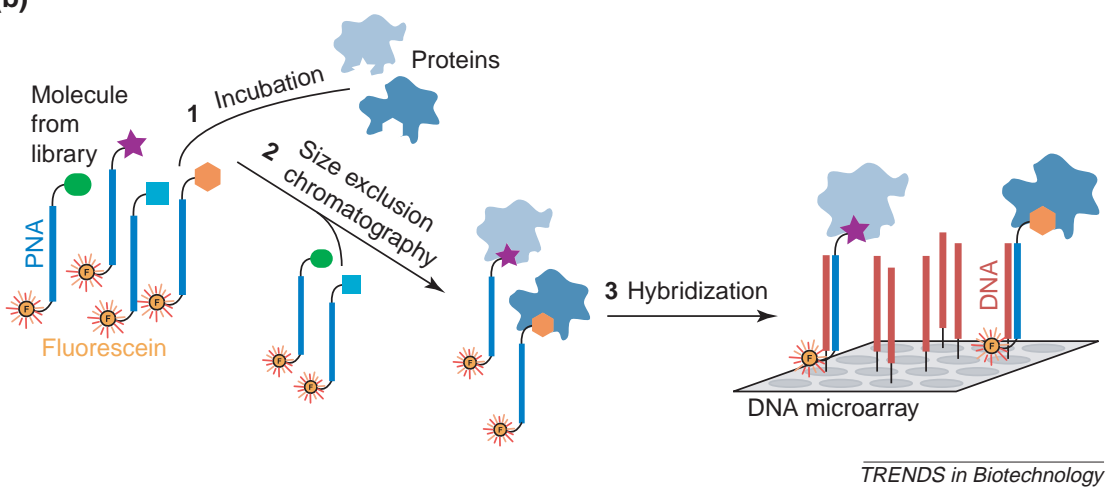

Figure 2. Analysis of labeled analytes. (a) Bead-based recovery of nucleic acids. Peptide nucleic acids (PNAs) are bound to beads with different internal labels. After hybridization with Alexa-532-labeled RNA, both beads and RNA are detected in a flow cytometer by two lasers, one for the bead and the other for the Alexa dye. (b) Molecule sorting on spatially addressable microarrays. PNAs with small molecules and fluorescein isothiocyanate (FITC) label are incubated with a protein mixture. After size exclusion chromatography, the protein-small-molecule-PNA complexes are hybridized to a microarray with cDNA oligonucleotides, encoding for the PNA tags. The signals of the FITC can be detected. 
Molecule sorting on spatially addressable microarrays PNA can also serve as a tag, encoding an attached molecule by a defined sequence. After a reaction or interaction between the tagged molecules in homogeneous solution, they are captured and thus separated on an array of nucleic acids that are of complementary sequence (Figure 2b). Winssinger et al. [21], for example, synthesized PNA-tagged small-molecule libraries. The libraries were synthesized on rink resin, using lysine as a branch molecule. The small molecules were coupled to the $\alpha$-amino groups of the lysines, the fluorescein-labeled PNA tags were attached at the $\varepsilon$-amino groups. These libraries were incubated with cell lysates. Subsequently, all library molecules that had not interacted with a protein from the cell lysates were removed by sizeexclusion filtration. The remaining molecular mixture was applied to an oligonucleotide microarray, which exhibited sequences that were complementary to the tags. The fluorescence signals produced at the various chip positions were indicative of the amount of protein that was present in the cell lysates and interacted with the respective small molecule. Even in crude cell lysates, different amounts of caspase-3 in apoptotic and nonapoptotic samples were clearly distinguishable [22]. The method could also be, to some extent, quantitative because there is only one label per PNA molecule, in contrast to common protein-labeling procedures, in which the number of labels varies because of the different numbers of reactive (e.g. thiol- or amino-) groups within each protein.

\section{Detection by means of labeled probe molecules}

Alternative to the introduction of a label into the analyzed sample, the probe molecule itself can also carry the reporter element. This has the advantage that the processing of the analyte is simplified, decreasing the degree of variability and reducing the effort required in sample preparation.

\section{Surface-attached molecular beacons}

Molecular beacons (Figure 3a) were first introduced by Tyagi and Kramer [23]. The molecules have a fluorescent dye at one terminus and a quencher molecule at the other. Without a hybridization partner, the conformation of a beacon is such that fluorophore and quencher lie next to each other and the molecule does not emit a signal. On hybridization, the molecule stretches out, separating the quencher and the fluorophore. Without the quenching effect, the fluorescence dye emits a signal, thus reporting the occurrence of hybridization. Molecular beacons represent a versatile tool in DNA diagnostics [24]. Although these molecules initially had a hairpin structure with a stem, it was later found, especially for PNA molecules, that a stem is not required for their functioning [25,26]. In contrast to DNA molecular beacons, stemless PNA beacons are less sensitive to ionic strength, and the quenched fluorescence of PNA is not affected by DNAbinding proteins. This enables the usage of PNA beacons under conditions that are not feasible for DNA beacons. Immobilization on both flat surfaces [27] and optical fibers [28] has been reported.

\section{Light-up probes}

Light-up probes (Figure $3 \mathrm{~b}$ ) are PNA molecules that could be used instead of molecular beacons, although this has not yet been demonstrated on solid supports. The PNA oligomer is labeled with a dye that is usually coupled to a flexible linker at the $\mathrm{N}$-terminal end. Upon binding to cDNA, the fluorescence quantum yield of the dye increases. With thiazole orange, for example, a fluorescence increase of $\sim 50$-fold was observed [29]. Whereas in DNA-oligonucleotides there is an electrostatic

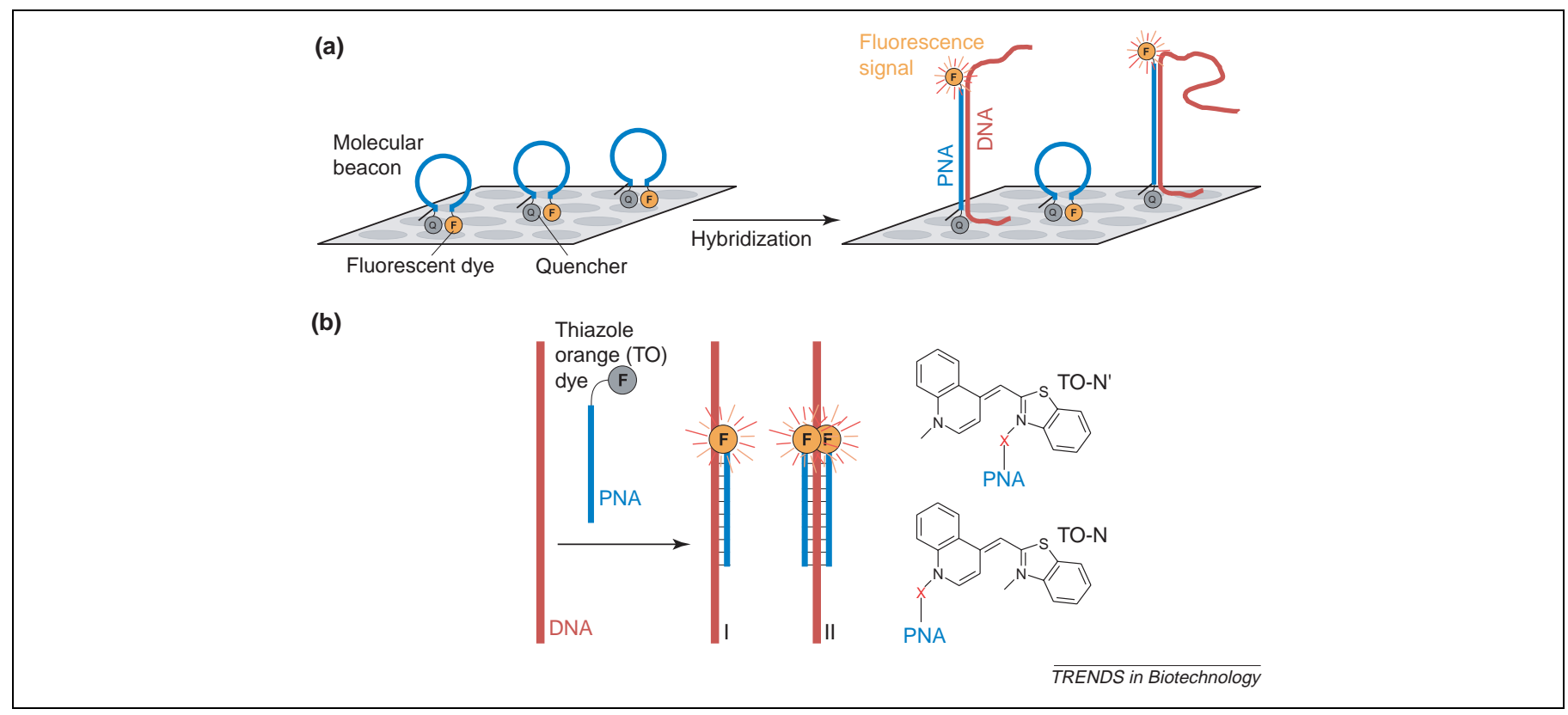

Figure 3. Detection by means of labeled probe molecules. (a) Surface-attached molecular beacons. The fluorescence signal of the surface-bound beacons is quenched. After

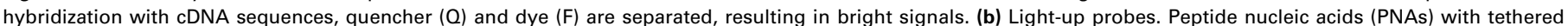

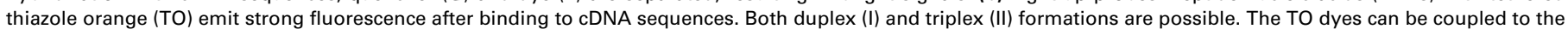
PNAs at different positions via a flexible linker (X). 
attraction between the negatively charged sugar-phosphate backbone and the cationic dye, this effect is avoided with the neutral PNA backbone. The PNA-based technique was used successfully for quantification of real-time PCR [30] and for the discrimination of target sequences that differ by a single base only [31].

\section{Detection with unlabeled probe and target molecules}

It would be advantageous to avoid labeling altogether, especially for routine applications. Besides speeding up the analytical process, all labeling steps introduce a bias because of differences in label incorporation. Moreover, direct electronic detection, for example, could assist in the integration of the assay into automated analysis systems, which are needed for high-throughput sample analysis.

\section{Surface plasmon resonance biosensors}

The hybridization of DNA or RNA targets to PNA-probes that are bound to planar surfaces can be recognized using surface plasmon resonance (SPR) (Figure 4a). Illuminated with polarized light, the refractive index of the array changes when DNA molecules hybridize to the PNAs on the sensor surface. The refractive index is directly proportional to the mass change on the chip surface. This method enables a real-time monitoring of the hybridization process during an experiment. In initial studies, the SPR technique was used to monitor the mass change during a continuous flow of oligonucleotides, which were either fully matched to the PNA or contained mismatched bases [32]. In a recent analysis, the variation in signal intensities for a single $\mathrm{C}$ to $\mathrm{T}$ conversion was reported to be as high as 300-400-fold [33]. However, to date, the sensitivity of the procedure has been inadequate for the analysis of complex mixtures. With oligonucleotides, concentrations of down to $0.2 \mu \mathrm{M}$ of an individual molecule could be detected.

Instead of using PNAs as immobilized probes and targets for free DNA, the opposite format was chosen by others [34]. Biotinylated PCR products were attached to the sensor surface and hybridized with PNA oligomers for the detection of point mutations in cystic fibrosis. It turned out that nonamer PNAs gave much better signals and were more discriminative than DNA oligonucleotides of 9 , 12 or 17 nucleotides in length. This might be because of the occurrence of secondary structures in the immobilized PCR products, which were more accessible to PNA probes than to DNA oligonucleotides. Successful analysis of normal, heterozygous and homozygous samples has been demonstrated. In further experiments, PNAs containing a 'chiral box' at their center showed higher mismatch recognition properties than normal molecules. The chirality was introduced by PNA monomers that were based on D-lysine instead of the usual $\mathrm{N}$-aminoethylglycine backbone [35-37].

\section{Time-of-flight-secondary ion mass spectrometry analysis of PNA microarrays}

A combination of PNA microarrays and time-of-flightsecondary ion mass spectrometry (TOF-SIMS) offers another possibility for the detection of unlabeled DNA targets [19,38] (Figure 4b). The method is very sensitive for detecting fragments $\left(\mathrm{PO}_{2}^{-}\right.$and $\left.\mathrm{PO}_{3}^{-}\right)$of the phosphate ions, which are an integral part of nucleic acids but are missing entirely in PNA. Subsequent to a hybridization of nucleic acids to a PNA microarray and the removal of nonspecifically bound molecules by appropriate washing steps, a primary ion beam is focused onto the microarray,

(b)

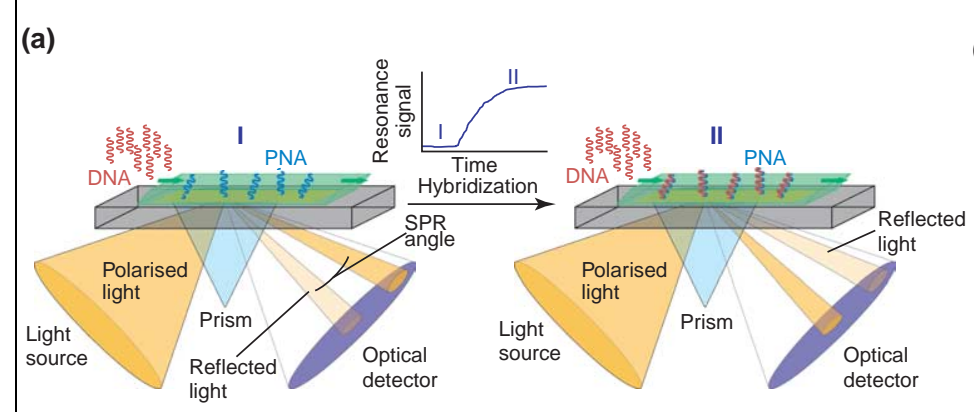

(c)

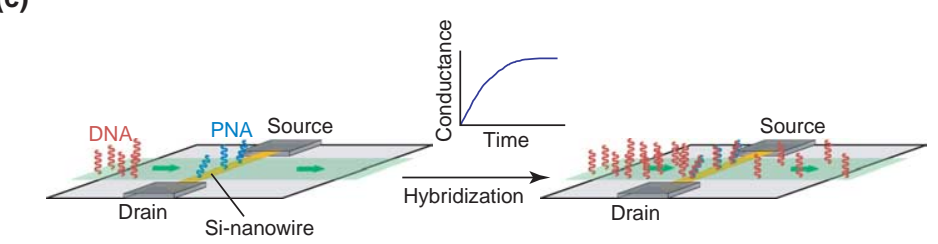

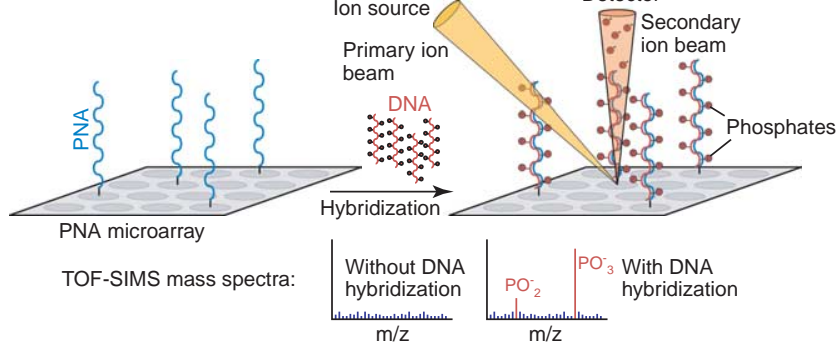

(d)

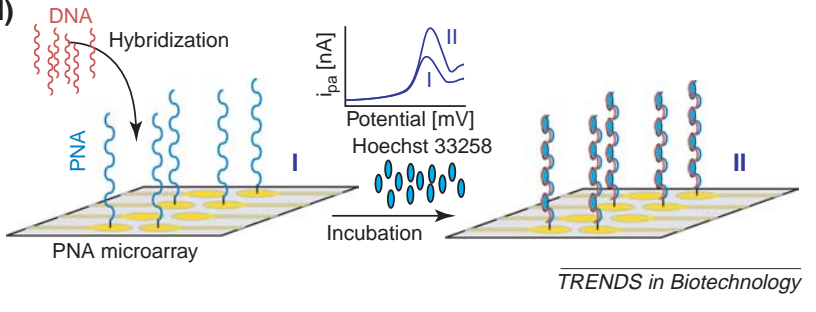

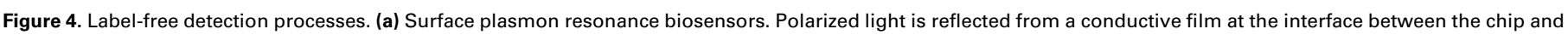

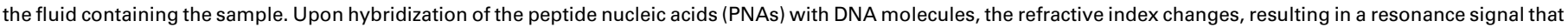

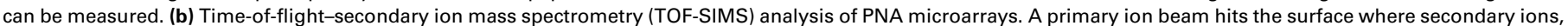

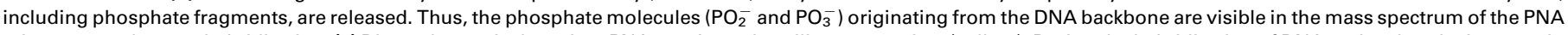

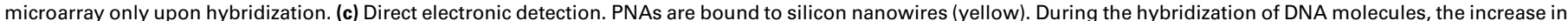

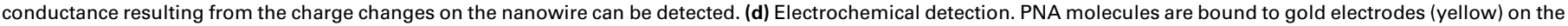

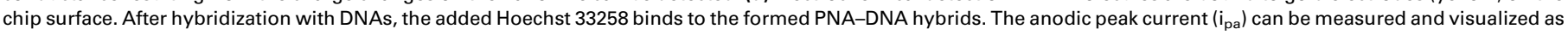
a linear sweep voltammogram. 
fragmenting a monolayer of molecules and releasing them from the surface as secondary ions. These are accelerated into a flight-tube, in which they are separated by their mass-to-charge ratios. Phosphates, and thus the respective signal, should only be present when a nucleic acid has bound to the complementary PNA molecule at a given position. For improved quality, the PNA molecules were covalently attached to the solid support via $\mathrm{N}$-terminal amino- or thiol-groups, using succinimidyl ester-activated, maleimide-activated or gold surfaces. Using this process, only full-length molecules were attached. Because of the extraordinary sensitivity of secondary ion mass spectrometry, it should be possible to detect the binding of genomic DNA directly. Thus, not only labeling but also the necessity for any prior amplification of the analyte by processes such as PCR could be avoided.

\section{Direct electronic detection}

Beside the obvious advantages of label-free detection, electrically addressable biosensors offer the potential of a much faster readout than is the case with current optical systems. Additionally, real-time measurement during the hybridization process would be possible. One step in this direction was the development of amine- and oxidefunctionalized silicon nanowires [39] (Figure 4c). Depositing PNA probes, Hahm and Lieber [40] were able to discriminate between wild-type and mutation sequences of the cystic fibrosis transmembrane receptor gene. The detection limit of the conductance measurements was $\sim 10 \mathrm{fM}$ of the DNA target [40]. In addition, initial encouraging results with impedance-based detection have been published recently [41]. Epoxysilane-modified and phosphorus-doped silicon $\left(\mathrm{Si} / \mathrm{SiO}_{2}\right)$ chips with covalently bound PNAs enabled the detection of changes in the charge distribution on the surface during the hybridization process. With 20-mer DNA oligonucleotides, good linear correlation of the out-ofphase impedance value and the DNA concentration was obtained in the range $0.1-2.0 \mathrm{ng} / \mu \mathrm{l}$.

\section{Electrochemical detection}

Various electrochemical detection methods have been investigated, based on the use of methylene blue [42], Tris (1,10-phenanthroline) cobalt (III) perchlorate [43] or Hoechst 33258 [44] as electrochemically active substances. Another option is the detection of oxidation signals from the guanine and adenine bases without any chemical additives [45]. In the first example, synthetic DNA oligomers were hybridized, and in the other two experiments small PCR products were used. Microfabricated PNA arrays were equipped with working electrodes $(\varnothing=200 \mu \mathrm{m})$ as well as counter and reference electrodes, all made of gold. Different PNA molecules having a cysteine at their $\mathrm{N}$-termini were bound to the electrodes via the thiol groups. The advantage of using PNAs is the weaker adhesion of the positively charged electrochemical compounds to the neutral backbone, thereby reducing the background signal. Hashimoto and Ishimori [44], for example, hybridized PCR products of the c-Ki-ras gene to such an array. After washing steps, the array was incubated in Hoechst 33258 solution. This solution binds to the minor groove of PNA-DNA duplexes. This produces an anodic peak current that is measured by linear sweep voltametry (Figure $4 \mathrm{~d}$ ). A detection threshold of $\sim 3 \mathrm{fmol}$ and 30 amol was found in hybridization experiments with dilutions of 20-mer DNA oligonucleotides and 128 base pair PCR products, respectively. The higher sensitivity of the analysis of PCR products results from the fact that more Hoechst 33258 binds to longer nucleic acids.

\section{Conclusion}

Although PNA has still not had quite the impact that was initially predicted, it is a DNA mimic that offers enormous potential for improved assay performance. Its main advantage is the combination of many features that are very similar to those of natural DNA with additional characteristics that are rather different. In view of the chemical and biological consequences of this hybrid status, PNA has a unique position compared with the many other nucleic acid derivatives, and could make an impact, particularly in the establishment of assay procedures for robust routine applications. Analyses with light-up probes or bead-based purification demonstrate the degree of improvement that is possible. Other methods would not be feasible without PNA; the TOF-SIMS detection of DNA hybridization is a good example of this. Although the uncharged backbone is limiting to some applications in solution, it causes no such problem on solid supports. However, PNA also exhibits a few disadvantages. A major one is the cost of the molecules. The high price, however, is mainly because of the relatively infrequent use of PNA. The basic components for the synthesis of PNA monomers should not be more expensive than those needed for nucleic acids. Therefore, the cost of synthesis should fall if the consumption of PNA molecules were to increase. In terms of specificity, unspecific binding events, such as in cases of polypurine sequences, have been reported [18]. However, this is no different to the behavior of DNA molecules of the same sequence but is made more apparent by the increased duplex stability. With current selection algorithms, such sequence bias can be avoided. Although there is still progress to be made, PNA offers an opportunity of advancing biosensor technology, especially with regard to sensitivity, automation and system integration.

\section{Acknowledgements}

We thank Anette Jacob for valuable discussions and her comments on the manuscript. The work of the authors was funded by the German Federal Ministry of Education and Research (BMBF) as part of the 'Leitprojekt Medizin', Nanotechnology and DHGP programs.

\section{References}

1 Nielsen, P.E. et al. (1991) Sequence-selective recognition of DNA by strand displacement with a thymine-substituted polyamide. Science 254, 1497-1500

2 Egholm, M. et al. (1993) PNA hybridizes to complementary oligonucleotides obeying the Watson-Crick hydrogen-bonding rules. Nature 365, 566-568

3 Igloi, G. (1998) Variability in the stability of DNA-peptide nucleic acid (PNA) single-base mismatched duplexes: real-time hybridization during affinity electrophoresis in PNA containing gels. Proc. Natl. Acad. Sci. U. S. A. 95, 8562-8567 
4 Schmidt, J.G. et al. (1996) Separation of 'uncharged' oligodeoxynucleotide analogs by anion-exchange chromatography at high $\mathrm{pH}$. Anal. Biochem. 235, 239-241

5 Demidov, V.V. et al. (1994) Stability of peptide nucleic acids in human serum and cellular extracts. Biochem. Pharmacol. 48, 1310-1313

6 Demidov, V.V. and Frank-Kamenetskii, M.D. (2004) Two sides of the coin: affinity and specificity of nucleic acid interactions. Trends Biochem. Sci. 29, 62-71

7 Uhlmann, E. et al. (1998) PNA: synthetic polyamide nucleic acids with unusual binding properties. Angew. Chem. Int. Ed. Engl. 37, 27962823

8 Nielsen, P.E. ed. (2004) Peptide Nucleic Acids: Protocols and Applications (2nd edn), Horizon Bioscience

9 Good, L. et al. (2000) Antisense PNA effects in Escherichia coli are limited by the outer-membrane LPS layer. Microbiology 146, 2665-2670

10 Demidov, V.V. (2001) PD-loop technology: PNA openers at work. Expert Rev. Mol. Diagn. 1, 343-351

11 Ørum, H. (2000) PCR clamping. Curr. Issues Mol. Biol. 2, 27-30

12 Murdock, D.G. and Wallace, D.C. (2002) PNA-mediated PCR clamping. Applications and methods. Methods Mol. Biol. 208, 145-164

13 Bockstahler, L.E. et al. (2002) Peptide nucleic acid probe detection of mutations in Mycobacterium tuberculosis genes associated with drug resistance. Biotechniques 32, 508-514

14 Komiyama, M. et al. (2003) PNA for one-base differentiating protection of DNA from nuclease and its use for SNPs detection. J. Am. Chem. Soc. 125, 3758-3762

15 Ren, B. et al. (2004) Straightforward detection of SNPs in doublestranded DNA by using exonuclease III/nuclease S1/PNA system. Nucleic Acids Res. 32, e42

16 Carlsson, C. et al. (1996) Screening for genetic mutations. Nature 380, 207

17 Igloi, G.L. (1999) Automated detection of point mutations by electrophoresis in peptide-nucleic acid-containing gels. Biotechniques $27,798-804$

18 Weiler, J. et al. (1997) Hybridisation based DNA screening on peptide nucleic acid (PNA) oligomer arrays. Nucleic Acids Res. 25, 2792-2799

19 Brandt, O. et al. (2003) PNA microarrays for hybridisation of unlabelled DNA samples. Nucleic Acids Res. 31, E119

20 Chandler, D.P. and Jarrell, A.E. (2003) Enhanced nucleic acid capture and flow cytometry detection with peptide nucleic acid probes and tunable-surface microparticles. Anal. Biochem. 312, 182-190

21 Winssinger, N. et al. (2001) From split-pool libraries to spatially addressable microarrays and its application to functional proteomic profiling. Angew. Chem. Int. Ed. Engl. 40, 3152-3155

22 Winssinger, N. et al. (2002) Profiling protein function with small molecule microarrays. Proc. Natl. Acad. Sci. U. S. A. 99, 11139-11144

23 Tyagi, S. and Kramer, F.A. (1996) Molecular beacons: probes that fluoresce upon hybridization. Nat. Biotechnol. 14, 303-308

24 Petersen, K. et al. (2004) Short PNA molecular beacons for real-time PCR allelic discrimination of single nucleotide polymorphisms. Mol. Cell. Probes 18, 117-122

25 Seitz, O. (2000) Solid-phase synthesis of doubly labeled peptide nucleic acids as probes for the real-time detection of hybridization. Angew. Chem. Int. Ed. Engl. 39, 3249-3252
26 Kuhn, H. et al. (2002) Hybridization of DNA and PNA molecular beacons to single-stranded and double-stranded DNA targets. J. Am. Chem. Soc. 124, 1097-1103

27 Wang, H. et al. (2002) Label-free hybridization detection of a single nucleotide mismatch by immobilization of molecular beacons on an agarose film. Nucleic Acids Res. 30, e61

28 Steemers, F.J. et al. (2000) Screening unlabeled DNA targets with randomly ordered fiber-optic gene arrays. Nat. Biotechnol. 18, 91-94

29 Svanvik, N. et al. (2000) Light-up probes: thiazole orange-conjugated peptide nucleic acid for detection of target nucleic acid in homogeneous solution. Anal. Biochem. 281, 26-35

30 Wolffs, P. et al. (2001) PNA-based light-up probes for real-time detection of sequence-specific PCR products. Biotechniques 31, 766-771

31 Isacsson, J. et al. (2000) Rapid and specific detection of PCR products using light-up probes. Mol. Cell. Probes 14, 321-328

32 Jensen, K.K. et al. (1997) Kinetics for hybridization of peptide nucleic acids (PNA) with DNA and RNA studied with the BIAcore technique. Biochemistry 36, 5072-5077

33 Burgener, M. et al. (2000) Synthesis of a stable and specific surface plasmon resonance biosensor surface employing covalently immobilized peptide nucleic acids. Bioconjug. Chem. 11, 749-754

34 Feriotto, G. et al. (2001) Peptide nucleic acids and biosensor technology for real-time detection of the cystic fibrosis W1282X mutation by surface plasmon resonance. Lab. Invest. 81, 1415-1427

35 Menchise, V. et al. (2003) Insights into peptide nucleic acid (PNA) structural features: the crystal structure of a D-lysine-based chiral PNA-DNA duplex. Proc. Natl. Acad. Sci. U. S. A. 100, 12021-12026

36 Sforza, S. et al. (2003) Direction control in DNA binding of chiral D-lysine-based peptide nucleic acid (PNA) probed by electrospray mass spectrometry. Chem. Commun. (Camb) 7, 1102-1103

37 Corradini, R. et al. (2004) Enhanced recognition of cystic fibrosis W1282X DNA point mutation by chiral peptide nucleic acid probes by a surface plasmon resonance biosensor. J. Mol. Recognit. 17, 76-84

38 Arlinghaus, H.F. et al. (1997) Analysis of biosensor chips for identification of nucleic acids. Anal. Chem. 69, 3747-3753

39 Cui, Y. et al. (2001) Nanowire nanosensors for highly sensitive and selective detection of biological and chemical species. Science 293, 1289-1292

$40 \mathrm{Hahm}$, J. and Lieber, C.M. (2003) Direct ultrasensitive electrical detection of DNA and DNA sequence variations using nanowire nanosensors. Nano Lett. 4, 51-54

41 Macanovic, A. et al. (2004) Impedance-based detection of DNA sequences using a silicon transducer with PNA as the probe layer. Nucleic Acids Res. 32, E20

42 Ozkan, D. et al. (2002) DNA and PNA sensing on mercury and carbon electrodes by using methylene blue as an electrochemical label. Bioelectrochemistry 58, 119-126

43 Wang, J. et al. (1997) Detection of point mutation in the p53 gene using a peptide nucleic acid biosensor. Anal. Chim. Acta 344, 111-118

44 Hashimoto, K. and Ishimori, Y. (2001) Preliminary evaluation of electrochemical PNA array for detection of single base mismatch mutations. Lab Chip 1, 61-63

45 Kara, P. et al. (2002) Label-free and label based electrochemical detection of hybridization by using methylene blue and peptide nucleic acid probes at chitosan modified carbon paste electrodes. Electroanalysis 14, 1685-1690 